\title{
Efeito da disponibilidade de hospedeiro e de alimento nas características biológicas de Trichogramma galloi Zucchi (Hymenoptera, Trichogrammatidae)
}

\author{
Dirceu Pratissoli ${ }^{1}$, Harley N. de Oliveira, ${ }^{1,3}$, Stella M. J. Vieira ${ }^{2}$, Regiane C. de Oliveira ${ }^{1,4}$ \& Hugo B. Zago ${ }^{1,4}$
}

${ }^{1}$ Centro de Ciências Agrárias, Universidade Federal do Espírito Santo. Alto Universitário s/nº, 29500-000 Alegre-ES, Brasil.

Endereço eletrônico: dirceu@npd.ufes.br

${ }^{2}$ Departamento de Engenharia Agrícola, Universidade Federal de Viçosa. 36571-000 Viçosa-MG, Brasil.

${ }^{3}$ Bolsista PROFIX/CNPq. Endereço eletrônico: hnoliveira@insecta.ufv.br

${ }^{4}$ Bolsista de Iniciação Científica PIBIC/CNPq.

\begin{abstract}
Efect of host and food availability on the biological characteristics of Trichogramma galloi Zucchi (Hymenoptera, Trichogrammatidae). Biological characteristics of Trichogramma galloi Zucchi, 1988 were evaluated in laboratory where these parasitoids were reared on eggs of Diatraea saccharalis (Lepidoptera, Pyralidae) with or without honey, and exposed to eggs of the host after $0,6,12,24,36,48,60,72$ and 84 hours of emergence. The parasitism rate and viability showed higher for individuals that received food. The sex ratio was not influenced by food. The number of individuals per egg only showed difference for those adults that did not receive food and stayed six hours without the host eggs. Checking the effect of the availability of eggs, only the sex ratio, with or without honey, did not show differences. The results show that $T$. galloi needs a carbohydrate supply and the time can influence the reproductive capacity.
\end{abstract}

Keywords. Biological control; food; host-parasitoid relationship; Trichogrammatidae.

Resumo. Características biológicas de Trichogramma galloi Zucchi, 1988 foram avaliadas em laboratório onde esses parasitóides foram criados com ovos de Diatraea saccharalis (Lepidoptera, Pyralidae), com ou sem mel, e expostos a ovos do hospedeiro após $0,6,12,24,36,48,60,72$ e 84 horas da emergência. As taxas de parasitismo e de viabilidade mostraram-se elevadas para indivíduos que receberam alimento. A razão sexual não foi influenciada pelo alimento. $\mathrm{O}$ número de indivíduos por ovo somente mostrou diferença para aqueles adultos que não receberam alimento e permaneceram 6 horas sem ovos do hospedeiro. Conferindo o efeito da disponibilidade de ovos, somente a razão sexual, com ou sem mel, não mostrou diferenças. Os resultados mostram que T. galloi necessita de um suprimento de carboidratos e que o tempo pode influenciar a capacidade reprodutiva.

Palavras-Chave. Controle biológico; relação hospedeiro-parasitóide; Trichogrammatidae.

A cana-de-açúcar tem como principal praga a broca Diatraea saccharalis (Fabricius, 1794) (Lepidoptera, Pyralidae) que, na fase larval, causa a morte da gema apical, o encurtamento dos entrenós, quebra da cana, enraizamento aéreo, germinação das gemas laterais e a perda de peso (MACEDo \& Botelho 1988).

Verificou-se que o estágio de ovo deve ser o principal alvo de combate (BотеLHо et al. 1995). Assim, parasitóides de ovos, principalmente do gênero Trichogramma, cujas espécies são abundantes no Brasil, são considerados uma alternativa viável (ZuCCHI \& MONTEIRO 1997).

O potencial de controle de D. saccharalis, empregando espécies de Trichogramma, tem sido estudado com o objetivo de obter todas as informações para que se possam criar programas de liberações inundativas. Entre as espécies desse parasitóide, Trichogramma galloi Zucchi 1988, é a mais visada, por possuir alta especificidade em relação àquela praga (ZuCCHI \& Monteiro 1997; Botelho 1997; Lima Filho \& Lima 2001).

Diversos fatores bióticos e abióticos podem interferir nas características biológicas desses parasitóides de ovos, levando-os a um comprometimento de sua eficiência no campo.
Dentre esses, o alimento tem interferência direta sobre a longevidade e fecundidade de espécies de Trichogramma (Berti \& Marcano 1991; McDougal \& Mills 1997). O tempo que a fêmea leva para encontrar seu hospedeiro também tem sido relacionado como um fator que interfere em sua capacidade reprodutiva (Berti \& MarCano 1991; Oliveira et al. 2000).

Como o alimento e o hospedeiro são fatores que podem comprometer a biologia e capacidade de busca desse parasitóide, este estudo objetivou simular uma condição de campo para avaliar o efeito do alimento e da disponibilidade de ovos do hospedeiro sobre o potencial de parasitismo, viabilidade, razão sexual e número de indivíduos por ovo de $T$. galloi.

\section{MATERIALE MÉTODOS}

Os ensaios foram conduzidos no laboratório de Entomologia do Centro de Ciências Agrárias da Universidade Federal do Espírito Santo, na cidade de Alegre, no Estado do Espírito Santo.

Para este estudo, foram utilizados exemplares de T. galloi 
coletados em ovos de D. saccharalis, no campus Dr. Leonel Miranda da Universidade Federal Rural do Rio de Janeiro, Rio de Janeiro.

Para manutenção e multiplicação de T. galloi, utilizou-se o hospedeiro alternativo Anagasta kuehniella (Zeller, 1879), seguindo-se a metodologia de PARRA (1997). Os ovos do hospedeiro alternativo foram colados em retângulos de cartolina azul celeste $(4 \times 2 \mathrm{~cm})$ com goma arábica diluída em água a $3 \%$. Posteriormente, esses cartões foram introduzidos em tubos de vidro $(8 \times 2,5 \mathrm{~cm})$, fechados com filme plástico de PVC, tendo em sua parede interna gotas de mel para alimentação dos adultos. Para que ocorra o parasitismo dos ovos, bem como evitar que haja superparasitismo, as fêmeas foram mantidas nos tubos por apenas 24 horas. Tanto a manutenção quanto a multiplicação desse parasitóide ocorreu em câmara climatizada regulada a $25 \pm 11^{\circ} \mathrm{C}$, umidade relativa de $70 \pm 10 \%$ e fotofase de 14 horas.

Para determinar a influência da ausência do hospedeiro por diferentes períodos de tempo $(0,6,12,24,36,48,60,72$ e 84 horas) e da presença ou ausência de alimento, fêmeas de $T$. galloi recém-emergidas foram isoladas em tubos de vidro (4,0 x $0,7 \mathrm{~cm}$ ), fechados com filme plástico de PVC. Para cada um dos intervalos, 30 fêmeas foram separadas, sendo que 15 delas receberam como alimento mel puro, e as demais permaneceram sem nenhum tipo de alimento. Cada fêmea dos dois tratamentos, a cada período de ausência do hospedeiro, foi exposta a uma cartela $(3,5 \times 0,5 \mathrm{~cm})$ contendo 40 ovos de $D$. saccharalis, com idade entre 0 e 12 horas. Os experimentos foram realizados nas mesmas condições usadas para a multiplicação dos parasitóides. O parasitismo foi permitido por 24 horas, sendo as cartelas retiradas dos tubos e acondicionadas em sacos plásticos $(23,0 \times 4,0 \mathrm{~cm})$, os quais foram fechados e mantidos nas mesmas condições até a emergência dos descendentes.

Os parâmetros avaliados foram os percentuais de parasitismo e de emergência de descendentes (viabilidade), a razão sexual, e o número de descendentes emergidos por ovo.

O delineamento utilizado foi inteiramente casualizado, com 15 repetições. Os dados foram submetidos à análise de variância e as médias comparadas pelo teste de Tukey a 5\% de probabilidade.

\section{RESULTADOS EDISCUSSÃO}

Fêmeas de T. galloi alimentadas, apresentaram maior taxa de parasitismo em ovos de $D$. saccharalis quando da ausência do hospedeiro por um período de 24 horas, com diferenças significativas nessa taxa, em relação àquelas que ficaram por mais de 48 horas sem receber ovos do hospedeiro; a menor taxa foi encontrada quando esse parasitóide recebeu ovos somente 84 horas após a emergência (Tabela I).

Para as fêmeas que não receberam alimento, constatou-se que a atividade de parasitismo ocorreu até 36 horas de ausência dos ovos do hospedeiro, com o maior percentual de parasitismo quando os parasitóides encontraram o hospedeiro imediatamente $(0 \mathrm{~h})$, com uma redução significativa nessa taxa ao longo do período de ausência (Tabela I).

Ao se comparar a percentagem de parasitismo entre fêmeas que receberam ou não o alimento, na faixa de 0 a 36 horas de ausência do hospedeiro, os valores foram estatisticamente superiores para as alimentadas, não sendo significativo apenas a 0 hora de disponibilidade dos ovos (Tabela I).

O percentual mais elevado de emergência dos descendentes (viabilidade), quando as progenitoras foram alimentadas, foi registrado no período de ausência do hospedeiro de até 12 horas, sendo estatisticamente significativo em relação aos demais períodos. O pior desempenho foi registrado quando as fêmeas permaneceram sem hospedeiro por 84 horas. Com relação às fêmeas que não receberam alimento, o maior percentual de emergência dos descendentes ocorreu quando T. galloi foi submetido a um período de 24 horas de ausência do hospedeiro, diferindo significativamente dos demais períodos (Tabela I).

Ao se comparar o percentual de emergência dos descendentes entre fêmeas alimentadas e não alimentadas, na faixa de 0 a 36 horas de ausência do hospedeiro, verificou-se que as alimentadas apresentaram valores significativamente superiores, com exceção das que foram submetidas a 24 horas de ausência (Tabela I).

Independente das fêmeas de $T$. galloi terem recebido ou não alimento, a razão sexual dos descendentes não mostrou diferenças estatísticas, independente do período em que as progenitoras foram submetidas à ausência de hospedeiro (Tabela I).

O número de indivíduos gerados por ovo, para fêmeas que receberam alimento e foram submetidas à ausência do hospedeiro por um período entre 0 e 6 horas, mostrou valores significativamente inferiores quando comparados com as que foram submetidas aos períodos de 24, 48 e 60 horas (Tabela I). Quando as fêmeas não receberam alimento mas foram imediatamente expostas ao hospedeiro, verificou-se que o número de descendentes gerados por ovo foi estatisticamente inferior em relação aos demais. No entanto, ao se comparar esse parâmetro entre fêmeas que receberam ou não alimento, verificou-se diferença estatística apenas quando as mesmas foram submetidas a 6 horas de ausência do hospedeiro (Tabela I).

Analisando os resultados, pode-se constatar que o suprimento alimentar foi o principal fator que interferiu em algumas das características biológicas de T. galloi. Confrontando esses dados com a bibliografia, verificou-se que BRowning \& MELTon (1987) ao compararem o parasitismo de oito espécies e/ou linhagens de Trichogramma em ovos de D. saccharalis, observaram grandes variações no número de ovos parasitados por fêmeas alimentadas. Nicoli (2002) observou que fêmeas de Trichogramma atopovirilia Oatman \& Platner 1983, quando alimentadas, apresentaram um parasitismo, em ovos de A. kuehniella, que variou entre 61,50 
Tabela I. Valores médios ( $\pm \mathrm{EP})$ da porcentagem de parasitismo, porcentagem de emergência (viabilidade), razão sexual e numero de indivíduos por ovo de T. galloi, submetidos à ausência de ovos de Diatraea saccharalis (hospedeiro) e de alimento (mel), por até 84 horas, em laboratório a $25 \pm 1^{\circ} \mathrm{C}, 70 \pm 10 \%$ e fotofase de 14 horas.

\begin{tabular}{|c|c|c|c|c|c|c|c|c|}
\hline \multirow{2}{*}{$\begin{array}{l}\text { Tempo } \\
\text { (horas) }\end{array}$} & \multicolumn{2}{|c|}{ Parasitismo(\%) } & \multicolumn{2}{|c|}{ Viabilidade(\%) } & \multicolumn{2}{|c|}{ Razão Sexual } & \multicolumn{2}{|c|}{$\mathrm{N}^{\circ}$ Ind/Ovo } \\
\hline & $\mathrm{C} / \mathrm{mel}$ & S/mel & $\mathrm{C} / \mathrm{mel}$ & S/mel & $\mathrm{C} / \mathrm{mel}$ & S/mel & $\mathrm{C} / \mathrm{mel}$ & S/mel \\
\hline 0 & $56,3 \pm 1.56 \mathrm{ABa}$ & $55,2 \pm 1.91 \mathrm{Aa}$ & $88,5 \pm 2.27 \mathrm{Aa}$ & $54,7 \pm 2.15 \mathrm{Bb}$ & $0,89 \pm 0.22 \mathrm{Aa}$ & $0,90 \pm 0.01 \mathrm{Aa}$ & $1,0 \pm 0.02 \mathrm{Ba}$ & $1,1 \pm 0.34 \mathrm{Ba}$ \\
\hline 6 & $52,5 \pm 1.26 \mathrm{ABa}$ & $44,2 \pm 2.92 \mathrm{Bb}$ & $91,5 \pm 1.00 \mathrm{Aa}$ & $54,8 \pm 1.51 \mathrm{Bb}$ & $0,85 \pm 0.02 \mathrm{Aa}$ & $0,91 \pm 0.02 \mathrm{Aa}$ & $1,0 \pm 0.00 \mathrm{Bb}$ & $1,5 \pm 0.05 \mathrm{Aa}$ \\
\hline 12 & $56,9 \pm 3086 \mathrm{ABa}$ & $33,8 \pm 3.21 \mathrm{Cb}$ & $88,8 \pm 1.76 \mathrm{Aa}$ & $51,3 \pm 2.37 \mathrm{Bb}$ & $0,87 \pm 0.02 \mathrm{Aa}$ & $0,95 \pm 0.01 \mathrm{Aa}$ & $1,4 \pm 0.05 \mathrm{ABa}$ & $1,5 \pm 0.03 \mathrm{Aa}$ \\
\hline 24 & $61,0 \pm 3.42 \mathrm{Aa}$ & $22,7 \pm 2.17 \mathrm{DEb}$ & $58,2 \pm 5.46 \mathrm{Bb}$ & $83,1 \pm 1.42 \mathrm{Aa}$ & $0,91 \pm 0.01 \mathrm{Aa}$ & $0,87 \pm 0.04 \mathrm{Aa}$ & $1,5 \pm 0.10 \mathrm{Aa}$ & $1,1 \pm 0.11 \mathrm{Aa}$ \\
\hline 36 & $56,9 \pm 2.72 \mathrm{ABa}$ & $16,9 \pm 0.59 \mathrm{~Eb}$ & $63,5 \pm 3.11 \mathrm{Ca}$ & $42,9 \pm 2.55 \mathrm{Bb}$ & $0,87 \pm 0.33 \mathrm{Aa}$ & $0,83 \pm 0.02 \mathrm{Aa}$ & $1,2 \pm 0.11 \mathrm{ABa}$ & $1,4 \pm 0.03 \mathrm{Aa}$ \\
\hline 48 & $47,8 \pm 2.08 \mathrm{~B}$ & - & $62,0 \pm 2.66 \mathrm{C}$ & - & $0,90 \pm 0.01 \mathrm{~A}$ & - & $1,5 \pm 0.11 \mathrm{~A}$ & - \\
\hline 60 & $50,8 \pm 1.99 \mathrm{~B}$ & - & $63,0 \pm 2.45 \mathrm{C}$ & - & $0,84 \pm 0.03 \mathrm{~A}$ & - & $1,5 \pm 0.06 \mathrm{~A}$ & - \\
\hline 72 & $46,7 \pm 2.94 \mathrm{~B}$ & - & $61,3 \pm 1.59 \mathrm{C}$ & - & $0,90 \pm 0.01 \mathrm{~A}$ & - & $1,4 \pm 0.08 \mathrm{AB}$ & - \\
\hline 84 & $36,4 \pm 1.35 \mathrm{C}$ & - & $50,0 \pm 3.23 \mathrm{D}$ & - & $0,86 \pm 0.01 \mathrm{~A}$ & - & $1,3 \pm 0.06 \mathrm{AB}$ & - \\
\hline
\end{tabular}

* Médias seguidas pela mesma letra maiúscula, na coluna, e letra minúscula, na linha, não diferem entre si pelo teste de Tukey a 5\% de probabilidade

e $94,75 \%$, e em fêmeas que não receberam alimento, o parasitismo variou de 58,75 a $72,91 \%$, o que, em ambas as condições, foi superior ao encontrado nesta pesquisa (Tabela I). HEGAZI et al. (2000) constataram que fêmeas sem alimento tiveram uma redução na atividade de parasitismo que variou de 10 a 26\%, nas primeiras 24 horas, para três espécies de Trichogramma. No entanto, resultado contraditório foi verificado por Santa-Cecília et al. (1987), que não encontraram diferença significativa no parasitismo de Trichogramma demoraesi Nagaraja 1983, quando as fêmeas receberam ou não mel como alimento.

Olson \& ANDOw (1998) e HegAzi et al. (2000) relataram que a presença ou não de alimento não interfere no percentual de emergência dos descendentes, o que também foi verificado por NiCOLI (2002), e que são discordantes dos resultados obtidos nesta pesquisa. Para a razão sexual, Leatemia et al. (1995) observaram que a presença e qualidade do alimento podem afetar a razão sexual, fato também verificado por Nicoli (2002) ao trabalhar com T. atopovirilia em ovos de A.kuehniella, contrariando os resultados encontrados no presente trabalho.

Um outro fator que também interferiu nas características biológicas foi o tempo que T. galloi leva para encontrar seu hospedeiro. Segundo Santa-Cecília et al. (1987), Fleury \& Bouletreau (1993) e Reznik et al. (1998), existe um decréscimo no parasitismo no decorrer do tempo de vida do parasitóide após a emergência, tanto para alimentados ou não, porém em intervalos maiores, conforme constatado nesta pesquisa. Concordando com esta afirmação, Resende \& Ciociola (1995) relataram que a maior taxa de parasitismo se dá nas primeiras 24 horas, com um total de 22 ovos de Helicoverpa zea (Boddie, 1850) parasitados por $T$. atopovirilia. Já Fleury \& Bouletreau (1993), mostram redução significativa do parasitismo de Trichogramma evanescens Weestwood, 1833 e Trichogramma brassicae Bezdenko, 1968, quando submetidos à ausência de hospedeiro por um período entre 24 e 96 horas. Este mesmo comportamento foi constatado por BERTI \& MARCANO (1991), trabalhando com Trichogramma pretiosum Riley, 1879, em ovos de Sitotroga cerealella (Olivier, 1819). Essas constatações são confirmadas pelos dados obtidos aqui, e podem ser explicados pelo fato que quanto maior o período de ausência do hospedeiro a que são submetidas as fêmeas, maior é a taxa de reabsorção de seus ovos (ORPHANIDEs \& GonZALEZ 1971; BERTI \& MARCANO 1991; REZNIK et al. 1998). Os resultados desta pesquisa referentes à viabilidade são contraditórios em relação aos de BERTI \& MARCANo (1991) e HARRISON et al. (1985), que verificaram que a viabilidade de Trichogramma não é afetada pelo tempo em que este ficou sem receber ovos para parasitar. Com relação à razão sexual, a não interferência do tempo de ausência do hospedeiro também foi constatada por BERTI \& MARCANO (1991) trabalhando com T. pretiosum sobre ovos de S. cerealella. No entanto, resultados divergentes foram encontrados por NICOLI (2002) para T. atopovirilia em ovos de A. kuehniella e S. cerealella.

Baseando-se nos dados desta pesquisa, bem como na revisão de literatura apresentada, pode-se concluir que Trichogramma necessita de uma fonte de carbohidratos, pois sua ausência interfere na fecundidade e na longevidade de $T$. galloi. O tempo de ausência do hospedeiro interfere na capacidade reprodutiva desse parasitóide de ovos, uma vez que espécies de Trichogramma são pró-ovigênicas, além de que essa ausência aumenta a taxa de retenção dos ovos nas fêmeas (Berti \& Marcano 1991; McDougall \& Mills 1997; Olson \& Andow 1998; Hegazi et al. 2000). As variações ocorridas nas características biológicas (parasitismo, viabilidade e número de indivíduos emergidos por ovo) podem estar relacionadas à diferença no potencial biótico das espécies e/ou linhagens de Trichogramma bem como em função de sua origem geográfica.

Como conclusão, pode-se dizer que a falta de alimento, bem como o tempo que as fêmeas de T. galloi gastam para encontrar seu hospedeiro, D. saccharaalis, podem interferir no seu potencial de colonização. 


\section{REFERÊNCIAS}

Berti, J. \& R. Marcano. 1991. Effect of time of host absence on parasitism by Trichogramma pretiosum Riley (Hym.: Trichogrammatidae). Boletin de Entomologia Venezolana 6(1): 5-10.

Botelho, P. S. M.; J. R. P. Parra; E. A. Magrini; M. L. Haddad \& L. C. L. Resende. 1995. Parasitismo de ovos de Diatraea saccharalis por Trichogramma galloi, em diferentes variedades de cana-de-açucar. Anais da Sociedade Entomológica do Brasil 24(1): 141-145.

Botelho, P. S. M. 1997. Eficiência de Trichogramma em campo, p. 303-318. In: J. R. P. PARRA \& R. A. ZuCCHI (eds.). Trichogramma e o Controle Biológico Aplicado. Piracicaba, FEALQ, xi + 324 p.

Browning, H. W. \& C. W. Melton. 1987. Indigenous and exotic Trichogrammatids (Hymenoptera: Trichogrammatidae) evaluated for biological control of Eoreuma loftini and Diatraea saccharalis (Lepidoptera: Pyralidae) borers on sugarcane. Environmental Entomology 16(2): 360-364.

FleURY, F. \& M. Bouletreau. 1993. Effects of temporary host deprivation on the reproductive potencial of Trichogramma brassicae. Entomologia Experimentalis et Applicata 68(3): 203-210.

Harrison, W. W.; E. G. King \& J. D. Ouzts. 1985. Development of Trichogramma exiguum and T. pretiosum at five temperature regimes. Environmental Entomology 14(2): 118 -121.

Hegazi, E. M.; W. E. Khafagi \& S. A. Hassan. 2000. Studies on three species of Trichogramma. I. Foraging behaviour for food or hosts. Journal of Applied Entomology 124(1): 145-149.

Leatemia, J. A.; J. E. Laing \& J. E Corrigan. 1995. Production of exclusively male progeny by mated, honey-fed Trichogramma minutum Riley (Hym.:Trichogrammatidae). Journal of Applied Entomology 119(8): 561-566.

Lima Filho, M. \& J. O. G. Lima. 2001. Massas de ovos de Diatraea saccharalis (Lep.: Pyralidae) em cana-de-açucar: Número de ovos e porcentagem de parasitismo por Trichogramma spp. (Hym.: Trichogrammatidae) em condições de laboratório. Neotropical Entomology 30(3): 483-488.

Macedo, N. \& P. S. M. Botelho. 1988. Controle integrado da broca da cana-de-açúcar Diatraea saccharallis. Brasil Açucareiro 106(2): $2-14$.

McDougal, S. J. \& N. J. Mills. 1997. The influence of hosts, temperature and food sources on the longevity of Trichogramma platneri. Entomologia Experimentalis et Applicata 83(2): 195-203.

Nicoli, E. M. 2002. Desenvolvimento e potencial de parasitismo de
Trichogramma atopovirilia (Hym.: Trichogrammatidae), sob a ausência de hospedeiro e de alimento. Dissertação de Mestrado. Vitória, Universidade Federal do Espírito Santo, 65 p.

Oliveira, H. N.; J. C. Zanuncio; D. Pratissoli \& I. Cruz. 2000. Parasitism rate and viability of Trichogramma maxacalii (Hym.: Trichogrammatidae) parasitoid of the Eucaliptus defoliator Euselasia apison (Lep.: Riodinidae), on eggs of Anagasta kuehniella (Lep.: Pyralidae). Forest Ecology Management 130 1-6.

Olson, D. M. \& D. A. Andow. 1998. Larval crowding and adult nutrition effects on longevity and fecundity of female Trichogramma nubilale (Hym.: Trichogrammatidae). Environmental Entomology 27(2): 508-514.

Orphanides, G. \& D. Gonzalez. 1971. Fertility and life table studies with Trichogramma pretiosum and T. retorridum (Hym.: Trichogrammatidae). Annals of the Entomological Society of America 64(4): 824-834

Parra, J. R. P. 1997. Técnicas de criação de Anagasta kuehniella, hospedeiro alternativo para produção de Trichogramma, p. 121150. In: J. R. P. PARRA \& R. A. ZUCCHI (eds.). Trichogramma e o Controle Biológico Aplicado. Piracicaba, FEALQ, xi + 324 p.

Resende, D. L. M. C. \& A. I. Ciociola. 1995. Capacidade de parasitismo de Trichogramma atopovirilia Oatman; Platner, 1983 (Hym.: Trichogrammatidae) em ovos de Helicoverpa zea (Boddie, 1850) (Lepidoptera: Noctuidae) em diferentes temperaturas. Ciência e Agrotecnologia 20: 421-424.

Reznik, S. Ya.; N. D. Voinovich \& T. Ya. Umarova. 1998. Egg retention in the presence of a host in Trichogramma females. Journal of Applied Entomology 122: 555-559.

SALMERON, E. 1989. Influência da geração de laboratório, temperatura e hospedeiro no parasitismo de duas espécies de Trichogramma (Hym.: Trichogrammatidae) coletadas em Diatraea saccharallis (Lep.: Pyralidae). Dissertação de Mestrado. Piracicaba, ESALQ/USP, $131 \mathrm{p}$.

Santa-cecília, L. V. C.; J. C. Matioli \& B. Sousa. 1987. Aspectos da biologia de Trichogramma demoraesi Nagaraja, 1983 e contribuições ao manejo de criações massais deste parasitóide em ovos de Anagasta kuehniella (Zeller, 1879). Anais da Escola Superior de Agricultura "Luiz de Queiroz" 44: 1607-1625.

Zucchi, R. A. \& R. C. Monteiro. 1997. O gênero Trichogramma na América do Sul. p. 41-66. In: J. R. P. PARRA \& R. A. Zucchi (eds.) Trichogramma e o Controle Biológico Aplicado. Piracicaba, FEALQ, xi + 324 p. 